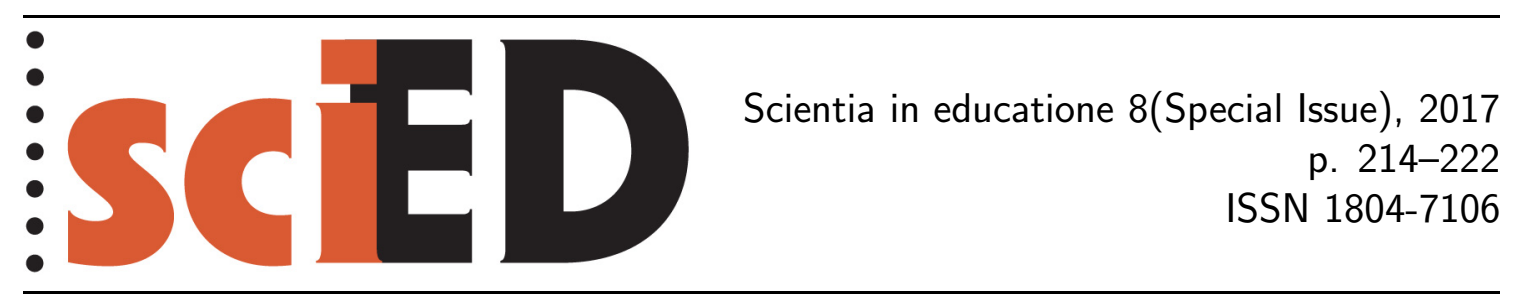

\title{
Exergy in School?
}

Tomaž Kranjc, Nada Razpet

\begin{abstract}
Students at all levels of physics instruction have difficulties dealing with energy, work and heat in general and, in particular, with the concepts of efficiency and ideal heat engine, and the maximum performance of refrigerators and heat pumps (Cochran \& Heron, 2006; Bucher, 1986). The reason for the difficulties is an insufficient understanding of the second law of thermodynamics (Kesidou \& Duit, 1992). In order to make these topics less difficult, the concept of exergy - well established as a powerful analytical tool in technical thermodynamics - describing the "quality" of energy, seems in our judgment to be worthy of inclusion in the physics curriculum at all levels. Its introduction does not add another law. It facilitates the understanding of irreversibilities (as the destruction of exergy) and gives a deeper meaning to the second law. In the treatment of heat engines the second-law efficiency throws a new light on the notions of an ideal and a real engine (similarly for a refrigerator or a heat pump). Exergy introduces, in a natural way, a distinction between various forms of energy according to its quality - availability for performing work. "Energy reserves", which can be better understood with the help of exergy, are of practical interest. From the thermodynamic point of view, a more correct term would be "availability reserves"; all around us, there are huge quantities of energy (in atmosphere, in oceans etc), but of very limited availability, i.e., of limited exergy.

In order to identify common misconceptions and difficulties encountered by students in the learning of the first and second law of thermodynamics, particularly in connection with heat engines and similar cyclic devices, we conducted a combined research among students of the Primary School Education at the Faculty of Education (UPR PeF) and of Biodiversity, Bioinformatics and Mediterranean Agriculture at the Faculty of Mathematics, Natural Sciences and Information Technologies (UPR FAMNIT) of the University of Primorska. Based on interviews and questionnaires given to two groups of students an experimental and a control group - in the beginning and the end of the semester, we investigated the influence (and possible advantages) of the introduction of the concept of exergy and the second-law efficiency.

In the presentation, we show a few examples that were treated with the experimental group in order to motivate the students and to make them familiar with the concept of exergy: the "energy losses" of a car engine and an analysis of improvements still allowed by nature; exergy loss associated with heat conduction; a simple exergy analysis of a heating house system (considering energy and exergy fluxes). We list some of the problems encountered by the students and the most common misconceptions as could be identified from the tests, questionnaires and interviews. An additional goal of the investigation is to test a longer-term knowledge of students.

From our research it would appear that exergy and the second-law efficiency are useful concepts which make it possible for students to get a better grasp of the material and to not only obtain a clearer understanding and knowledge of standard topics like heat engines, but also a broader view and insight into the meaning of energy and both the first and the second law, and their interrelation.
\end{abstract}

Key words: heat, exergy, second-law efficiency. 


\section{INTRODUCTION}

In physics instruction, the chapter on heat is considered difficult and abstract, despite its usefulness and broad applications in technology. For the understanding of the laws of thermodynamics one needs to know and understand the concepts of work, heat, energy, and entropy, and develop an intuitive feeling for them. Heat engines and similar cyclic devices (refrigerators, heat pumps, gas turbines, fuel cells, rocket engines, etc.) are important examples of technology based on thermodynamics and, in fact, one of the goals of teaching thermodynamics is "an appreciation of the limits to efficiency" (Baierlein, 1994). The school physics instruction is usually limited to heat engines, refrigerators, and heat pumps (Arnaud, Chusseau \& Philippe, 2010; Bartlett, 1976; Leff \& Teeters, 1977; Tobin, 1969). Their efficiency (or a suitable measure of their performance) is introduced and calculated and a comparison is made to the efficiency of ideal (Carnot) engines. For engines using (the ideal) gas as their working substance the efficiency can be determined by a direct calculation of cyclic changes. But it is more important for students to understand the limits of the functioning of heat devices as imposed by the laws of thermodynamics.

In physics instruction at the high school level or in university programs that include an introductory course of physics for non-physics majors (e.g. chemistry, biology, mathematics, etc. majors) one again and again sees that students have not learned certain simple facts and/or have not understood them. In order to achieve better results numerous methods were developed and applied (Baierlein, 1994; Tobin, 1969; Cochran \& Heron, 2006; Das, 1994; Finfgeld \& Machlup, 1960; Leff \& Teeters, 1977; Marcella, 1992; Reif, 1999; Reynolds, 1994; Samiullah, 2007; Kesidou \& Duit, 1992). They were supposed to help gain a better understanding and a higher level of competency in applying the laws and methods of thermodynamics.

In view of a small success of these methods and efforts, we join proposals aiming at introducing the concept of exergy into the instruction of thermodynamics (from the elementary school level on) (see Viglietta, 1990), together with related notions and quantities. We believe that, based on the concept of exergy and the second-law efficiency, students can better understand and memorize the functioning and the underlying principles of heat devices and, at the same time, largely extend their understanding of some relevant topics of the present days. Besides, a study of a much broader spectrum of devices, device parts, and processes is made possible.

\section{HeAt ENGINES AND STUDENT UNDERSTANDING}

Among the main instructional tools illustrating cyclic changes repeated by heat engines and similar devices are diagrams of heat flows and work. Schematic presentations of heat flows and work (as in Figure 1) are easy to read but students often do not see connections to real engines - they do not know where the system (heat engine, refrigerator, heat pump) is "hidden" or where in the real device are the heat reservoirs.

An additional problem occurring with this kind of presentation of a heat device is the fact that it is not obvious from it how the supplied heat divides between the produced work and exiting heat. This is not determined by the energy but by the entropy law which has to be additionally built into the diagram.

Cochran and Heron (2006) assessed the knowledge and understanding of the second law among different groups of students and presented the responses to questions 
that they posed based on heat flow and work diagrams (Figure 1). Students received three different diagrams (for a heat-engine, refrigerator and a "strange device", Figure 1); for each of them they had to tell if such a device could function and explain why they thought so. The results obtained at final exams gave only about $30 \%$ correct answers.

Testing second year students of the Primary school Education at the Faculty of Education (UPR PeF) and Biodiversity, Bioinformatics and Mediterranean Agriculture at the Faculty of Mathematics, Natural Sciences and Information Technologies (UPR FAMNIT) of the University of Primorska (in the academic year 2011/2012) we obtained similar results as in (Cochran \& Heron, 2006) (between $30 \%$ and $40 \%$ correct answers).

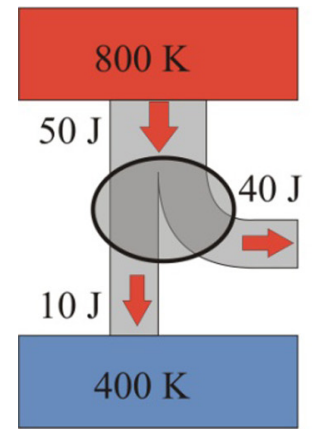

a)

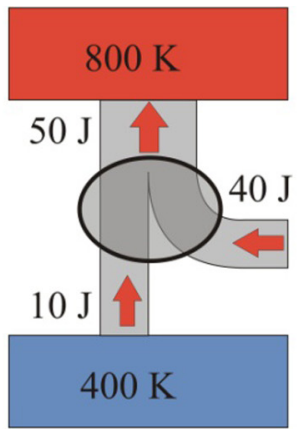

b)

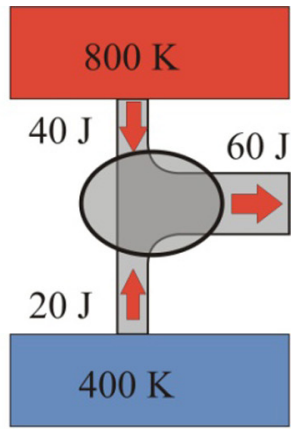

c)

Figure 1: Heat devices for test questions (Ref. (Cochran \& Heron, 2006)). a) A proposed heat engine, b) a proposed refrigerator, c) a "strange device". Students had to determine if the devices could function and why. (Figure 1 in Ref. (Cochran \& Heron, 2006))

Three additional questions were about the "ideal" heat engine for which it was repeatedly emphasized in class to be a synonym for the Carnot heat engine. The first question asked for the efficiency of an ideal heat engine, the second, what would be the efficiency of an imaginary ideal heat engine operating between the extreme temperatures of $300 \mathrm{~K}$ and $299 \mathrm{~K}$ (the example was solved in the class), for the third question students had to draw the heat and work flow diagram of an ideal engine.

The portions of correct answers were as follows. The first question: $35 \%$, the second question: $44 \%$, the third question: $21 \%$, the total number of students: 34 . 53 percent (18 students) gave $100 \%$ as the answer to the first question (i.e. they wrote that the efficiency of the ideal heat engine is $100 \%$ ). It is interesting that none of the students who claimed the efficiency of an ideal heat engine to be $100 \%$ drew as the answer to question 3 the diagram on the right of Figure 2, which would be a logically consistent answer. $62 \%$ drew approximately the diagram in Figure 2 left (with the line showing the flow of emitted $Q_{L}$ being more or less thin), obviously suggesting that the ideal heat engine directs "almost all" the received heat into work.

It is obvious that students are not really able to use the second law when they think about cyclic heat devices. Bucher (1986) proposed and other authors (Wallingford, 1989; Yan \& Chen, 1990, 1992; Bucher, 1993; 2007; Wallingford, 1989; Yan \& Chen, 1990, 1992) subsequently further developed a new type of diagrams (Bucher diagrams), which include both the first and the second law of thermodynamics. However, the new diagrams do not seem to have enough appeal, visualizing force and simplicity to be adopted in school curriculum.

In presenting any study material, the choice of basic concepts is of upmost importance. In the treatment of thermodynamic systems such a new quantity could be 

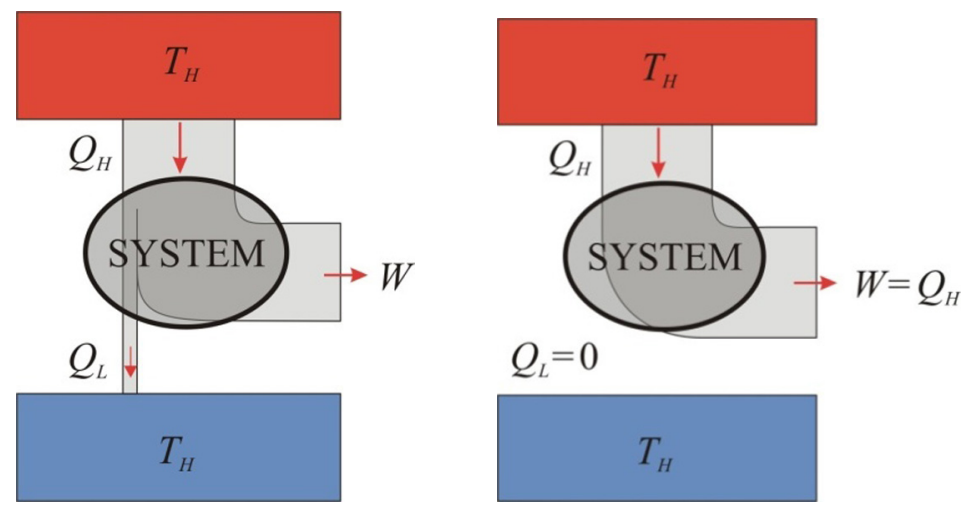

Figure 2: Left: Flow of heat and work in an ideal heat engine as shown by a majority of students. Right: None of the students who answered that the efficiency of the ideal heat engine is $100 \%$ drew a diagram with $Q_{L}=0$, which would correspond to $100 \%$ efficiency

exergy (Rant, 1956). Not only it allows a more reliable analysis of heat engines and similar devices, but also offers a better insight into the role of energy of arbitrary processes and into the very understanding of energy and its uses. It could be characterized as a concept for the valuation of the quality of energy. We believe it to be a useful, effective and at the same time a sufficiently simple concept and therefore appropriate to be introduced in a sensible way into the school physics curriculum.

\section{EXERGY AND THE SECOND LAW-EFFICIENCY}

Energy has two facets, quantity and quality. The first law of thermodynamics states that energy is preserved, i.e., that it "cannot be destroyed or come out of nothing". Energy appears in many different forms, like kinetic, potential, elastic, electric, chemical, atomic, thermal, etc., and it can change from one form into another. Regardless of the processes and transformations the amount of energy stays the same.

Since energy is preserved we really should not be talking about "energy losses". However, energy that is preserved in its amount and is therefore not lost does not have "the same value" in every form. In all real processes its "quality" decreases. For example, possibilities of using the (potential) energy of a weight hanging above the ground are greater than possibilities for using its internal energy coming from the change of the potential energy when the weight falls on the ground and warms up a little bit.

Let us call exergy the quantity which expresses the quality of energy. We can say that exergy $\left(E^{k}\right)$ is the energy that can be, in given circumstances, transformed into an arbitrary other form of energy. We often say that exergy is the part of energy that can be used for work in its entirety or, represents the available work. Exergy is therefore the "useful" part of energy. The remaining - useless part - is called anergy $\left(E^{a}\right)$. The entire energy $(E)$ can be written as $E=E^{k}+E^{a}$.

Different forms of energy can then be divided into three classes, depending on the "quality":

- Energy that can be completely used for work= exergy

- Energy that can be partly used for work=exergy + anergy

- Energy that cannot be transformed into work= anergy 
Mechanical, electric and (approximately) chemical energy can transform into work in its entirety (if we ignore irreversibilities which are part of any real process and are a consequence of different dissipation processes like friction). In the case of an electric engine the electric work can be (almost) completely transformed into the mechanical work. The thermal energy can be (partly) transformed into work in the case where the system is not (yet) in equilibrium with its environment.

What is the exergy of a certain amount of heat $\left(Q_{H}\right)$ that can be taken from a heat reservoir at temperature $T_{H}$ ? A Carnot heat engine is the most efficient device for converting heat into work. The exergy of heat $Q_{H}$ is the portion which is available for work and this is $|W|=Q_{H}\left(1-T_{0} / T_{H}\right)$ ( $T_{0}$ being the lower reservoir temperature). The partition of $Q_{H}$ into exergy and anergy is therefore $Q_{H}=|W|+Q_{H}\left(T_{0} / T_{H}\right)$.

This result shows that exergy is not an "absolute" quantity depending only on the quantity of invested energy but is also dependent on circumstances: it depends on the temperatures at which a Carnot heat engine absorbs and emits heat. The efficiency $\eta_{C}=1-T_{L} / T_{H}$ is greater if the temperature at which the Carnot engine emits heat is lower. This is often (though not always) the temperature of the environment $T_{0}$ $\left(T_{L}=T_{0}\right)$.

Efficiency tells us what limitations for converting heat into work are imposed by nature under given circumstances $\left(T_{0}\right.$ and $T_{H}$, say). For given $T_{0}$ and $T_{H}$ the work $\left(\left|W_{\max }\right|\right)$ that can be obtained from heat $Q$ in the best case scenario (reversibility of the process) equals $\left|W_{\max }\right|=\eta_{C} Q$. In the hypothetical example where $T_{0}=299 \mathrm{~K}$ and $T_{H}=300 \mathrm{~K}, \eta_{C}$ would equal $1 / 300$ or about $0.3 \%$. Even though this is not much it is the most allowed by nature in given circumstances. Therefore this is the "ideal" efficiency of an "ideal" heat engine.

Due to unavoidable irreversibility of real processes and also for other reasons the work $\left(\left|W_{\text {real }}\right|\right)$ is actually smaller than the maximum $\left(\left|W_{\max }\right|\right)$ and the same holds true for the actual efficiency $(\eta), \eta<\eta_{C}$. Therefore, it seems reasonable to compare the actual efficiency of a device with the maximum possible efficiency. To do that we introduce the second-law efficiency of a heat engine $(\nu)$ (Backhaus \& Schlichting, 1984) as $\nu=\eta / \eta_{C}$. In the "ideal" case, $\nu=1$.

The information about the second-law efficiency of a heat engine is important because it tells us what the "reserves" are when one gets work out of heat. If $\nu=1$, there is no room left for any improvement, even though the efficiency $\eta$ might be small. If, however, $\nu<1$, nature still allows improvements in the engine's efficiency.

Let us formulate the Second Law of Thermodynamics in terms of exergy instead of entropy:

- Exergy is preserved under all reversible changes.

- In irreversible transformations exergy decreases ("exergy losses") and changes into anergy.

\section{INTRODUCING EXERGY INTO THE TEACHING OF THERMODYNAMICS}

In school year 2012/2013 we again tested second-year students of the Primary school Education at the UPR PeF) and Biodiversity, Bioinformatics and Mediterranean Agriculture at the UPR FAMNIT.

Students were divided into two groups. With one of them we used the standard approach to cover the chapter on heat engines, refrigerators, and heat pumps 
(without introducing exergy). With the other one we introduced exergy and, besides the standard thermal efficiency for heat engines $\left(\eta=|W| / Q_{H}\right)$ or coefficient of performance $(\mathrm{COP})$ for refrigerators $\left(Q_{L} /|W|\right)$ and heat pumps $\left(Q_{H} /|W|\right)$ we also introduced the second-law efficiency, $\nu=\eta / \eta_{C}$. The second group, according to our observations, obtained a better insight into the understanding of energy, its consumption and uses, and with that of energy issues in general (which implicitly includes the issues concerning ecology). In testing, however, we were interested how the students from the second group were able to answer questions which belong to the standard coverage of thermodynamics.

The whole group had 43 students who were divided into two groups, the first one (for the standard approach) had 21 students, the second (for the introduction of exergy) had 22 students. The obtained results (Figure 3 ) show a convincingly better answers of the second group to the first three questions. It appears that through the treatment of heat devices with the help of exergy they obtained a better insight and feeling for the content of especially the second law of thermodynamics.

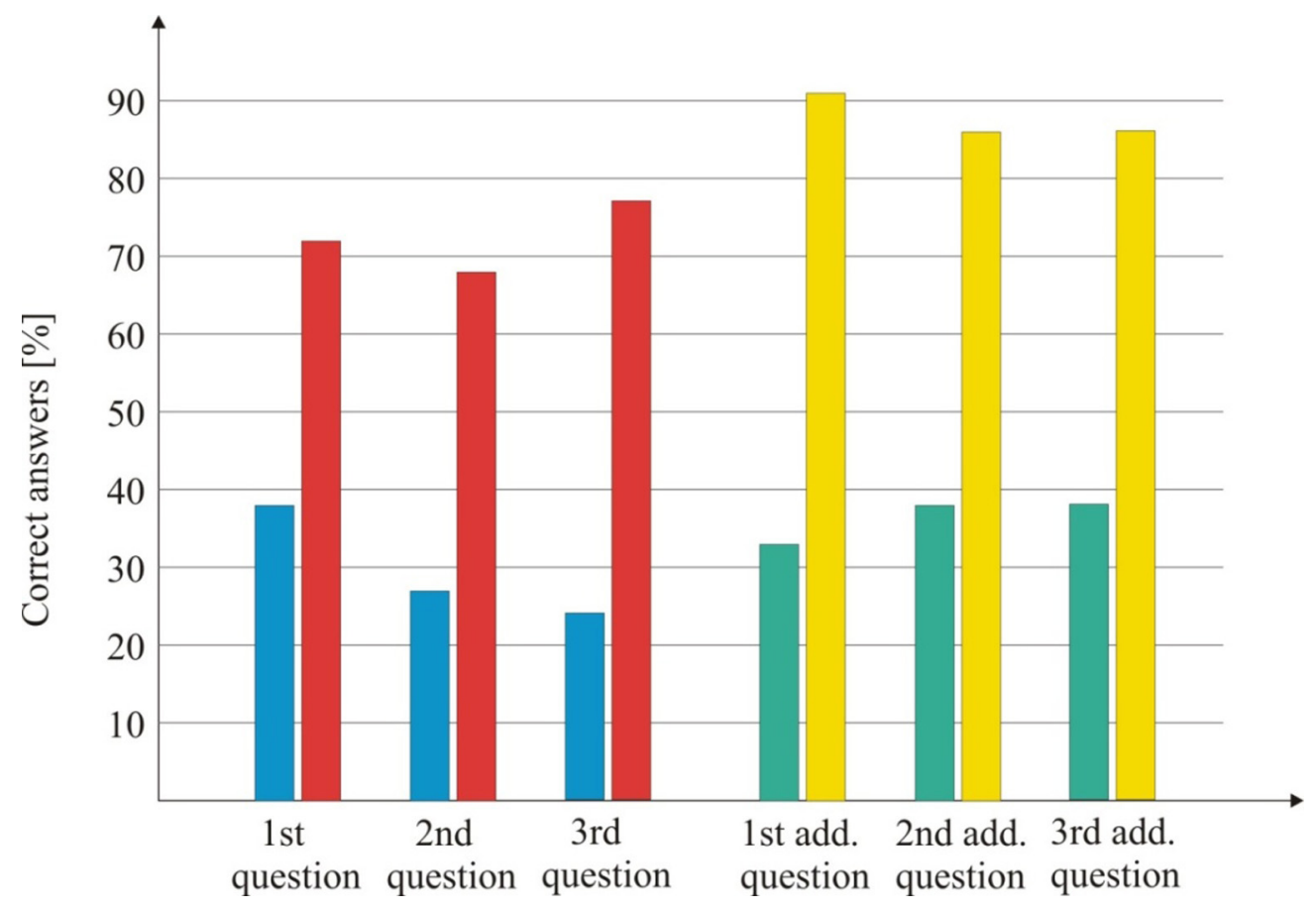

Figure 3: The correct answers to questions from Fig. 1 and to the three additional questions. The first column of each question refers to the first group, the second to the second group

Also with the three additional questions about the "ideal" heat engine (cf. the text after Figure 1) the second group did much better. The results are shown in Figure 3. The example from the second question was not solved in class this time, and in question 3 they had to add an explanation of the diagram that they drew.

\section{CONCLUSIONS}

Thermodynamics is both an abstract (and for this reason difficult) and a technologically important chapter of physics. It is therefore worth making an effort to acquaint students with some basic concepts and ideas and also with the simplest information 
about technical applications. Among these the most important in school are cyclic heat devices (heat engines, refrigerators, heat pumps).

Even though it is difficult to introduce innovations in the time when the scope of physics instruction is diminishing at all levels, we believe that after several decades (Rant, 1956) or even more of a successful introduction especially in the field of technical thermodynamics it is reasonable to think about introducing the concept of exergy into the physics curriculum at all levels.

It appears that with the help of exergy it is possible to better understand the First and the Second Law of Thermodynamics and the limitations of transforming heat into work set by nature. At the same time one can better understand where it is possible to further improve one's devices (where nature still allows it) and what is true the meaning of exploitation of energy and energy reserves.

After instruction of thermodynamics based on the concept of exergy, students have

- showed a better understanding of the significance of the two laws of thermodynamics,

- got to know and appreciate the concept of the quality of energy,

- acquired a better insight into the restraints put by the second law on natural processes,

- arrived at a better understanding of reversible and irreversible processes in terms of exergy losses,

- got to understand much better cyclic devices (heat engines, refrigerators, heat pumps),

- got to understand the meaning of "ideal" devices in terms of the 1st law efficiency and the 2nd law efficiency,

- were able to do simple energy-exergy analyses,

- arrived to understand better terms like "energy reserves", "energy crisis", "energy degradation", "waste of energy", "lost work", "renewable energy sources", "availability" etc., and to build a better attitude toward ecological issues connected to energy needs.

With the use of exergy it was demonstrated at least on the experimental groups of students that the exergy concept helped them to a better understanding of the material which already is a part of the existing school curriculum and has to be mastered by students.

It is our opinion that the introduction of the concept of exergy leads to a better and deeper students' understanding and insight of the fundamental laws of thermodynamics as well as of their use in many technical and social applications. At the same time, it does not require changes of the curriculum (but the introduction of a new concept), it requires no extra time and no increase in the study input. Therefore we expect a serious discussion and consideration about a suitable introduction of exergy into the classroom instruction. 


\section{REFERENCES}

Arnaud, J., Chusseau, L. \& Philippe, F. (2010). A simple model for Carnot heat engines. Am. J. Phys., 78(1), 106-110.

Backhaus, U. \& Schlichting, H. J. (1984). Der exergetische Wirkungsgrad. Der Physikunterricht, 18(3), 58-61.

Baierlein, R. (1994). Entropy and the second law: A pedagogical alternative. Am. J. Phys., 62(1), 15-26.

Bartlett, A. A. (1976). Introductory experiment to determine the thermodynamic efficiency of a household refrigerator. Am. J. Phys., 44(6), 555-559.

Bucher, M. (1986). New diagram for heat flows and work in a Carnot cycle. Am. J. Phys., 54(9), 850-851.

Bucher, M. (1993). Diagram of the second law of thermodynamics. Am. J. Phys. 61(5), 462-466.

Bucher, M. (2007). Comment on "Development and assessment of research-based tutorials on heat engines and the second law of thermodynamics", by Matthew, J. Cochran, Paula, R. L. Heron. Am. J. Phys., 75(4), 377-379.

Cochran, M. J., \& Heron, P. R. L. (2006). Development and assessment of research-based tutorials on heat engines and the second law of thermodynamics. Am. J. Phys., 74(8), $734-741$.

Das, S. K. (1994). Comment on "Entropy production and the second law of thermodynamics: An introduction to second law analysis", by Thomas V. Marcella. Am. J. Phys., 62(12), 1151-1152.

Finfgeld, C. \& Machlup, S. (1960). Well-Informed Heat Engine: Efficiency and Maximum Power. Am. J. Phys., 28(4), 324-326.

Kesidou, S. \& Duit, R. (1992). Students' conceptions of the second law of thermodynamics: An interpretative study. J. Res. Sci. Teach., 30, 85-106.

Leff, H. S. \& Teeters, W. D. (1977). EER, COP, and the second law efficiency for air conditioners. Am. J. Phys., 46(1), 19-22.

Marcella, T. V. (1992). Entropy production and the second law of thermodynamics: An introduction to second law analysis. Am. J. Phys., 60(10), 888-895.

Rant, Z. (1956). Exergie, ein neues Wort für 'Technische Arbeitsfähigkeit'. Forsch. Ing. Wes., 22, 36-37.

Reif, F. (1999). Thermal physics in the introductory course: Why and how to teach it from a unified atomic perspective. Am. J. Phys., 67, 1051-1062.

Reynolds, R. E. (1994). Comment on "Entropy production and the second law of thermodynamics: An introduction to second law analysis," by Thomas V. Marcella. Am. J. Phys., 62(1), 92.

Samiullah, M. (2007). What is a reversible process? Am. J. Phys. 75(7), 608-609.

Tobin, M. C. (1969). Engine efficiencies and the second law of thermodynamics. Am. J. Phys., 37(11), 1115-1117.

Viglietta, L. (1990). 'Efficiency' in the teaching of energy. Phys. Educ., 25, 317-321. 
Wallingford, J. (1989). Inefficiency and irreversibility in the Bucher diagram. Am. J. Phys., 57(4), 379-381.

Yan, Z. \& Chen, J. (1990). Modified Bucher diagrams for heat flows and works in two classes of cycles. Am. J. Phys., 58(4), 404-405.

Yan, Z. \& Chen, J. (1992). New Bucher diagrams for a class of irreversible Carnot cycles. Am. J. Phys., 60(5), 475-476.

TOMAŽ KRANJC

NADA RAZPET

Faculty of Education, University of Ljubljana, Ljubljana, Slovenia

Faculty of Education, University of Primorska, Koper, Slovenia 